\title{
EVOLUTION IN THE NUCLEUS
}

A review of David Allen's research into the nature of the Galactic Centre

\author{
M.G. BURTON \\ University of New South Wales \\ School of Physics \\ Sydney, NSW 2052 \\ Australia
}

\begin{abstract}
A personal reflection is presented of David Allen's research into the content of our Galactic nucleus.
\end{abstract}

\section{Introduction}

David Allen's contributions to astronomy are legend, and encompassed a depth and breadth that is staggering. From public populariser to innovative researcher, David's achievements were exceptional at every level, through virtually every field of astronomy, from planetary atmospheres to quasar evolution. A few themes, however, coursed especially strongly through his work. One of these was the nature of our Galactic nucleus. I have had the great privilege to work closely with David during the last four years of his work in this arena, and would like to reflect upon some of the insights we have gleaned as a result of his perception.

Two intertwined threads run through his work, the evolution in IR detector technology and the evolution in ideas. During a decade where IR instruments evolved from single element bolometers, through simple linear arrays, to the sophisticated array detectors now available, David developed the IRPS, the FIGS and finally IRIS for use on the Anglo Australian Telescope. He used these in novel ways to extract just that information of interest from the vast data stream pouring into the telescope. Through its interpretation we witnessed an evolution of ideas about the core of the Galaxy. They moved from that of an ailing nucleus dominated by old giants surrounding a massive black hole centred on IRS 16, to that of a youthful, vigorous nucleus, the product of a mild starburst, and perhaps only a low mass, passive black hole, in its core. 
David Allen's bibliography contains over 500 articles, and those on the Galactic Centre are listed at the end of this article. This review will attempt to summarise their achievements. David, of course, would never claim the credit for this work on his own, and fully acknowledged the contributions of his co-workers. He would also pay close regard to the work of others in the field, and allow their ideas to influence his work. I am afraid I am unable to adequately attribute all the credit that David would give to his colleagues here. This is not an historical treatise, and a proper treatment of the evolution of our understanding of the Galactic centre would naturally provide a more comprehensive review of the contributors to the field. But as all who worked with David well knew, he was the driving force behind it, whatever the field.

\section{The Evolution in Observational Technique}

David's first instrument in the 80's was the IRPS (Infrared Photometer Spectrometer), a single channel instrument with broad band filters, and a $1 \%$ CVF for low resolution spectroscopy. With the genius of John Barton's electronics this could work in simple DC mode, averting the need for spatial chopping, a facet that David quickly realised would allow him to readily map IR sources. Accumulating data as the telescope scanned rapidly back and forth across a source, complete with allowance for telescope inertia, the maps obtained were both unprecedently deep and had better spatial integrity than any other group was achieving [eg. 2, 3, 5]. For instruments of its type, the IRPS was never beaten. Though it has been far surpassed by the technology now available, it gave David great satisfaction in his final months to know that the IRPS was still being put to good use. It had been redeployed to the South Pole, and was spending the winter sitting outside at $-60^{\circ} \mathrm{C}$, site testing the Antarctic Plateau for a future IR observatory!

By the middle of the decade a linear array was available, and FIGS (the Fabry-Perot Infrared Grating Spectrometer) was born. Though FIGS was never quite the success it was originally hoped to be, it still produced its fair share of exciting new science. David's innovation was an extension of DC imaging, to develop imaging spectroscopy. However this time the chopping secondary was scanned across a source, instead of the telescope. Readouts were synchronised to the scan speed, and a 3D data cube built up, containing 2 spatial and one spectral dimension [eg. 6, 11].

Finally in the 90's the AAO obtained an infrared array, and with the help of Peter Gillingham's brilliant optical design, David came up with IRIS (the Infrared Imaging Spectrometer, though David will insist that there is no acronym, simply a name), an all-singing, all-dancing IR camera / spectrometer. The innovative use this time was again drift scanning, but 
now slowly drifting the spectrometer slit across the source to build the data cube. The entire $\mathrm{H}(1.6 \mu \mathrm{m})$ and $\mathrm{K}(2.2 \mu \mathrm{m})$ bands could be measured simultaneously [eg. 8, 9, 10,12,13,14,15,16], and spectral maps at any desired wavelength obtained from the resultant cube. It might perhaps be noted, though, that the full data reduction, involving correcting for the tiny tracking inaccuracies of the telescope over a 2 hour scan, could be a little tedious at times!

\section{The Evolution in Ideas}

Perhaps surprisingly, David's first venture in the field of the Galactic Centre $[1,4]$ in fact involved a discovery and then a retraction! But it was an error he freely admitted to. An L'-band $(3.8 \mu \mathrm{m})$ spectrum towards IRS7 revealed a broad absorption. In particular, there were several discrete features in the absorption trough. It was speculated that their origin might indicate the presence of complex organic molecules in the interstellar grains. However, follow up work with the UKIRT telescope later showed no evidence for these features! David eventually convinced himself that he had been the victim of a rather obscure systematic error, the result of the light path of the standard and source passing through a slightly different part of the CVF on the IRPS.

The next piece of research moved to the nature of the black hole presumed to lurk at the centre of the Galaxy. A sub-arcsecond 'image', obtained via scanning with the IRPS [2], revealed new details in the central parsec. First, was the obvious presence of some regions of particularly heavy obscuration, presumed to signal the presence of small molecular clouds. We now easily recognise these as the circumnuclear ring. Second, there was the surface brightness profile, shown to continue as an $\mathrm{r}^{-0.7}$ power law to 1 " from the (presumed) centre. This did not conform to an isothermal model $\left(\mathrm{r}^{-1.0}\right)$, unless the core was much smaller than that of any globular cluster known. Any core radius would have to be $<2$ " or $<0.08 \mathrm{pc}$. It suggested to him a density cusp, with a massive central object. To account for $10^{7} \mathrm{M}_{\odot}$ in the central parsec, as deduced from [NeII] kinematics (Lacy et al., 1980), implied a core density of $>10^{8} \mathrm{M}_{\odot} \mathrm{pc}^{-3}$.

A companion paper [3] with John Storey took this work further. A $1 \mu \mathrm{m}$ CCD image, plus $\mathrm{J}(1.2 \mu \mathrm{m})$ and $\mathrm{K}$ band maps, were used to determine stellar types from colours, and to achieve better astrometric precision between the radio position for $\mathrm{SgrA}^{*}$ and the IR image. IRS16 was resolved into 3 components, with the designated IRS16-C $1.0 \pm 0.4$ " $\mathrm{E}$ from the best astrometric position they could determine for $\operatorname{SgrA}^{*}$. While with hindsight we can see that this difference is significant, so strong was the belief that SgrA* must be associated with a stellar-like source, they concluded IRS16- 
$\mathrm{C}$ represented the centre of the Galaxy. Making this identification allowed the cusp-like distribution inferred in [2] to be extended to within 0.6 " of the centre, strengthening the case for a massive black hole. From its colour, significantly 'bluer' than the M-supergiant IRS7, the mean spectral type was estimated to be around mid-K. Thus, from the flux of IRS16-C they deduced that approximately 100 giants inhabited the 1 " region around the nucleus. Storey and Allen must have had an inkling that all might not be right with this conclusion, however, for they very wisely finished off their paper with a recommendation that improved astrometry be obtained to better tie together the radio and infrared reference frames!

David's next contribution to the Galactic Centre was the one that really made his mark on the field, and has a controversy still raging today. With Bob Saunders he indeed obtained the better data needed above. They asked the question in the title of a provocative Nature paper "the galactic centre black hole: only a dwarf?" [5]. Having identified common features in L' and $\mathrm{M}(4.8 \mu \mathrm{m})$ band images to the radio 6-cm image of Lo \& Claussen (1983), it was clear that $\mathrm{SgrA}^{*}$ was over 1" W of IRS16-C. While in agreement with the earlier result in [4], this time it was certain the offset was for real.

The implications were considerable. A faint IR source was associated with SgrA*, but now the complex structure around IRS16 could no longer be identified as the core of a cusp around the central object. Any black hole associated with SgrA* must have low luminosity. To provide the UV energy required to heat the dust and produce the far-IR emission would have needed a $\mathrm{K}$ band flux at least 20 times greater. Thus SgrA* could not be providing the bulk of the luminosity from the nucleus. This then raised the question of the source of the ionizing radiation? Allen \& Saunders argued that the $M$ supergiants present implied a burst of star formation about $10^{7}$ years ago, leaving a concentration of B stars there now. They thus concluded that around $100 \mathrm{~B}$ stars made up IRS16, split into the four components then identified, which together provided the luminosity for the Galactic centre. However, this raised a severe problem for the massive black hole model. The proximity of the IRS16 'clumps' to SgrA* would result in their dispersion within a few hundred years, unless star formation was continuously occurring. But the tidal field of a massive black hole would inhibit the latter process. Allen \& Saunders provided us with a coherent picture; an isothermal core with radius $0.1 \mathrm{pc}$ and central stellar density $>10^{7} \mathrm{M}_{\odot} \mathrm{pc}^{-3}$, a few small associations of newly formed B stars within that core, and an accreting black hole with a mass of no more than 100 $\mathrm{M}_{\odot}$ •

The next piece of work $[6,11]$ developed this idea further. On the scene came FIGS, and the ability to obtain spectral images. A spectral cube in $\operatorname{Br} \alpha(4.05 \mu \mathrm{m})$ showed both broad and narrow components to the line, with 
striking changes in only $1.5 "$ " The broad component, over $1000 \mathrm{~km} / \mathrm{s}$ in extent, was likened to the profiles seen in Wolf-Rayet stars. Images in and outside the $\mathrm{CO}$ bandhead absorption features (at $2.3 \mu \mathrm{m}$ ) revealed a bimodal population of hot (no absorption) and cool (with absorption) stars. The hot star distribution was strongly peaked on IRS16. It was concluded this represented a population of early-type stars, whereas the cool stars were $M$ giants and supergiants. The most interesting feature in the spectral cube, however, turned out to be that of HeI $(2.06 \mu \mathrm{m})$, where a single bright star (the 'AHH' star) was apparent. With a profile similar to the broad hydrogen lines, and a strength twice that of the $2.16 \mu \mathrm{m} \mathrm{Br} \gamma$ line, such a spectrum had only been seen before in supergiants classified as WN9 and Ofpe in the Magellanic Clouds (McGregor, Hillier \& Hyland, 1988). Finally, an unidentified line was discovered at $2.22 \mu \mathrm{m}$, confined to the radio 'minicavity' 3" to the SW of SgrA*. This has only recently been identified as due to [Fe III] (Lutz et al., 1994). Folding all this into the picture, their conclusion was that a burst of star formation occurred in the Galactic Centre a few million years ago, leading today to the hot, evolved wolfrayet-type $\mathrm{AHH}$ star, and a cluster of 10-100 $\mathrm{B}_{[\mathrm{e}]}$ stars associated with IRS16. With temperatures $\sim 30,000 \mathrm{~K}$, luminosities in the range $10^{5-6} \mathrm{~L}_{\odot}$ and masses in the range $20-60 \mathrm{M}_{\odot}$, together they account for virtually all the UV and bolometric luminosity of the central parsec. They suggested our galaxy be regarded as a weak starburst.

Technology development marched on, and in the early 90 's the AAO finally obtained a Rockwell IR array detector. IRIS was built to house it, and a new camera turned to the Galactic centre. Through drift scan imaging, 3D data cubes were again built up containing the entire $\mathrm{K}$-band spectrum. They were both considerably more extensive, had better spatial resolution, and went much deeper, than the FIGS variety. David was first involved in some perhaps incidental work before he resumed the study of the stellar population. Better images of the $\mathrm{H}_{2}(2.12 \mu \mathrm{m})$ and $\mathrm{Br} \gamma$ lines were obtained $[8,13]$, illustrating the hot molecular gas in the circumnuclear disk and showing a close correspondence between the IR and radio recombination lines (but not exact, which lead an interesting study by Roberts and Goss (1993) on the 3D geometry). The spectra lead some support to the PDR model for the excitation of the molecular ring, and helped rule out a hot, ionized bar. On the discovery of a transient radio source, IRIS was used to search for a stellar counterpart [7]. Curiously, the transient appeared in the middle of one of the dense clouds of the circumnuclear ring, leading to speculation of a SN. However no source was identified, and the limits placed on its IR luminosity made such a scenario look unlikely.

The delay in receiving an array meant that David missed being the actual discoverer of the cluster of He I stars (Krabbe et al., 1991), and the 
demonstration that these could provide the UV to power the $10^{7} \mathrm{~L}_{\odot}$ far-IR luminosity from the central parsec. But IRIS soon confirmed this result [8], and quickly extended the range of stellar types that make up the young cluster through images in the line of [Fe II] $1.64 \mu \mathrm{m}$ [13] and by extending the technique of hot and cool star separation previously demonstrated with the FIGS $[10,16]$. Some 30 or so hot emission line stars were identified in the Galactic core from their $\mathrm{HeI}, \mathrm{Br} \gamma,[\mathrm{FeII}]$ and/or lack of $\mathrm{CO}$ bandhead emission. They are surrounded by the ionized gas of the radio 'mini-spiral', also seen clearly in the $\mathrm{Br} \gamma$ image. This ionized gas abutts the inside edge of a hot, clumpy molecular ring, seen in the light of $\mathrm{H}_{2}$ [8].

Most intriguing, however, was David's last piece of analysis, from the hot and cool star separation [10,14]. It revealed that the hot, population I stars formed a tight cluster, with a very much more compact core than that of the population II stars of the Galactic bulge, seen in the cool star picture. The comparison with Eckart et al's (1993) speckle data was fascinating. They saw 350 individual sources in the inner parsec, contributing at least $90 \%$ of the flux there, and each brighter than $\mathrm{K} \sim 14$. These sources also formed a tight core, of radius about 4 " $(0.2 \mathrm{pc})$, and their absolute absolute magnitude required them to be either $\mathrm{M}$ giants or $\mathrm{OB}$ stars. The flux distribution of hot stars was virtually identical to the number distribution of Eckart et al. David concluded that they represented the same population.

The implications were considerable. It would imply these 350 stars were essentially all OB stars; young, massive stars that only recently formed, with the most massive just starting to evolve off the main sequence. They form a population I core to the centre of the Galaxy, with a combined mass in the range $10^{3-4} \mathrm{M}_{\odot}$. They would not, however, dominate the mass in the centre. This would be either from the cool stars, or from a massive central object. But the comparison could be taken further. From the $\sim 100 \mathrm{~km} / \mathrm{s}$ velocity dispersion seen in CO (Sellgren et al., 1990), and the 20" (1 pc) core radius of the cool star flux distribution, a mass density of $10^{6} \mathrm{M}_{\odot} \mathrm{pc}^{-3}$ is derived for the inner parsec, similar to that obtained from dynamical arguments of the gas motions. If $\operatorname{SgrA}^{*}$ were to weigh $10^{6} \mathrm{M}_{\odot}$ then the central parsec would be virtually devoid of stars (other than the 350 seen by Eckart et al.). The (simpler) alternative is that there is no large central object, and the combined mass of the cool, bulge stars dominates.

This conclusion is, to say the least, controversial! Its validity depends on whether the flux and number distributions can indeed be equated. Already some evidence has been presented at this conference by Reinhard Genzel to suggest that this might not be the case. If not, and the core radius really is as small as 4", then the central mass density would rise to $\sim 10^{8} \mathrm{M}_{\odot} \mathrm{pc}^{-3}$, easily accommodating the requirements of a massive black hole. 
While the argument over the central mass continues, the evidence for a starburst grows. David used IRIS to explore selected regions in the central 50 parsecs $[9,12,15]$, initially to seek an ionization source for the arched radio filaments. Instead, a number of new star clusters were discovered, and spectroscopic imaging has shown they also contain similar emission line stars as those in the nucleus. Not as many, but still suggesting a recent episode of massive star formation, which has now left $\mathrm{WN}$ and $\mathrm{B}_{[\mathrm{e}]}$ stars. The conditions in these clusters are not extreme, and no other reasonable explanation other than star formation has been given for their presence. It suggests the conditions of the central parsec are not unique, and thus calls into question explanations other than star formation for the massive stars found there.

\section{Conclusions}

I have tried to describe David Allen's immense contributions to the debate on the Galactic nucleus, though any such account can only be incomplete. Although David knew his own picture would surely evolve as observations continued to improve, he left us believing he had given us a view which was both self-consistent and simple. It is one of life's tragedies that he will no longer be able to respond to the latest data, and develop his own theories to accommodate them. I would like to use David's own words to summarise his picture, as presented in his own unique style at the last conference he attended, a workshop on the Galactic Centre held at Sydney University in November 1993.

"The Galactic centre is a retirement village where old population II stars go to live out their closing years. But we see a second, younger population in the central parsec. Why? A group of young stars formed there $\sim 10^{7}$ years ago, presumably as the result of a molecular cloud collision. Dynamical interactions have pushed the population II stars outwards. In other words, when the hooligans arrived the old folk feared for their well being and moved out!"

And David leaves us with two questions:

- Why the small spread in mass and spectral type?

- Is the dynamical timescale adequate?

To end, I would like to pass on two pieces of wisdom that David was fond of telling us about. The first was the principle of Ockham's Razor. Now that $\mathrm{SgrA}^{*}$ is no longer at the centre of the light distribution, nor is needed to account for the luminosity and the mass loss rate, why do we still need to use it account for the mass (since this is why a large central mass was first postulated)? The second is that the inner cluster, for which we now observe 
such complex structure, would only subtend 0.1 " even at the distance of the nearby Andromeda Galaxy. Yet we cannot claim to understand its origin. Surely therein lies cautionary advice for active galaxy pundits.

\section{Bibliography}

\subsection{PAPERS IN REFEREED JOURNALS}

Allen, D. A. and Wickramasinghe, D. T. (1981) 'Diffuse interstellar absorption bands between 2.9 and $4.0 \mu \mathrm{m}^{\prime}$, Nature, 294, 239. [1]

Allen, D. A., Hyland, A. R. and Jones, T. J. (1983) 'High-resolution images of the Galactic centre', Mon. Not. R. Astr. Soc., 204, 1145. [2]

Storey, J. W. V. and Allen, D. A. (1983) 'The Galactic nucleus', Mon. Not. R. Astr. Soc., 204, 1153. [3]

Jones, T. J., Hyland, A. R. and Allen, D. A. (1983) ' $3 \mu \mathrm{m}$ spectroscopy of IRS 7 towards the Galactic centre', Mon. Not. R. Astr. Soc., 205, 187. [4]

Allen, D. A. and Sanders, R. H. (1986) 'The Galactic centre black hole: only a dwarf?' Nature, 319, 191. [5]

Allen, D. A., Hyland, A. R. and Hillier, D. J. (1990) 'The source of luminosity at the Galactic centre'. Mon. Not. $R$. astr. Soc., 244, 706. [6]

Zhao, J-H., Roberts, D. A., Goss, W. M., Frail, D. A., Lo, K. Y., Subrahmanyan, R., Kesteven, M. J., Ekers, R. D., Allen, D. A., Burton, M. G. and Spyromilio, J. (1992) 'A transient radio source near the center of the Milky Way Galaxy', Science, 255, 1538. [7] Burton, M. G. and Allen, D. A. (1992) 'Imaging the hot molecular gas at the centre of the Galaxy', Proc. Astr. Soc. Aust., 10, 55. [8]

Cotera, A. S., Erickson, E. F., Allen, D.A., Colgan, S. W. J., Simpson, J. P., and Burton, M. G. (1994) 'A cluster of hot stars near the galactic center arched filaments', Astrophys. $J .$, in preparation. [9]

Allen, D. A. and Burton, M. G. (1994) 'The population I core of the Galaxy', Proc. Astr. Soc. Aust., 11, 191. [10]

\subsection{PAPERS IN CONFERENCE PROCEEDINGS}

Allen, D. A., Hyland, A. R., Hillier, D. J. and Bailey, J. A. (1989) 'The stars that power the Galactic centre', Int. Astr. Union Symp., 136, 513. [11]

Cotera, A. S., Erickson, E. F., Simpson, J. P., Colgan, S. W. J., Allen, D. A. and Burton, M. G. (1992) 'A new cluster of hot stars near the Galactic center', Bull. Amer. Astr. Soc. 24, 1262, ABSTRACT. [12]

Burton, M. G. and Allen, D. A. (1993) 'Near-IR imaging spectroscopy of the hot gas in the centre of the Galaxy'. In Astronomical Infrared Spectroscopy: Future Observational Directions, ed. S. Kwok, Astr. Soc. Pacif. Conf. Ser., 41, 289. [13]

Allen, D.A. (1994) 'The central stellar cluster', Ringsberg conference on The Galactic Centre, in press. [14]

Cotera, A. S., Erickson, E. F., Allen, D.A., Colgan, S. W. J., Simpson, J. P., and Burton, M. G. (1994) 'The discovery of hot stars in the vicinity of the thermal filaments', Ringsberg conference on The Galactic Centre, in press. [15]

Burton, M.G. and Allen, D.A. (1994) 'The population I core at the centre of the Galaxy', Proc. Airborne Astronomy Symp. on the Galactic Ecosystem: From Gas to Stars to Dust, ed. M. R. Haas, J. A. Davidson and E. F. Erickson, (San Francisco: ASP), in press. [16] 


\subsection{POPULAR ARTICLES}

Allen, D. A. (1983) 'The heart of our Galaxy', Yearbook of Astronomy 1984, 147. [17] Allen, D. A. (1993) 'What lurks at the centre of our Galaxy?', Yearbook of Astronomy $1994,125 .[18]$

\section{References}

Eckart, A., Genzel, R., Hofmann, B., Sams, B.J and Tacconini-Garman, L.E. (1993) Ap. J. Lett., 407, L77.

Lacy, J.H., Townes, C.H., Geballe, T.R. and Hollenbach, D.J. (1980) Ap. J., 241, 132. Lo, K.Y. and Claussen, M.J. (1983) Nature, 306, 647.

Lutz, D., Krabbe, A. and Genzel, R. (1994) Ap. J., in press.

Krabbe, A., Genzel, R., Drapatz, S. and Rotaciuc, V. (1991) Ap. J. Lett., 382, L19.

McGregor, P.J., Hillier, D.J. and Hyland, A.R. (1988) Ap. J., 334, 639.

Roberts, D.A. and Goss, W.M. (1993) Ap. J. Supp., 86, 133.

Sellgren, K., McGinn, M.J., Becklin, E.E. and Hall, D.N.B. (1990) Ap. J., 359, 112. 


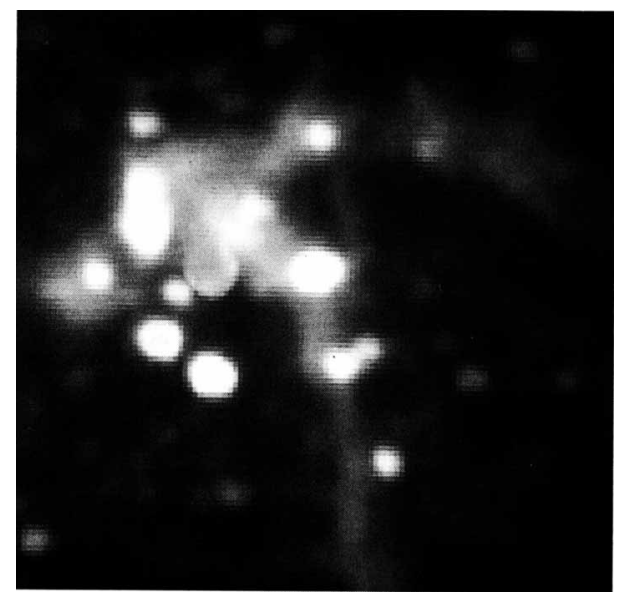

Figure The IRPS $3.8 \mu \mathrm{m}$ image (from [5]) in blue, overlaid on the 6-cm radio continuum image of Lo \& Claussen (1983) in red, tying together the radio and infrared reference frames via the emission from IRS $2 / 13$, seen in both frames. This image demonstrated clearly that IRS16-C (which is not priminent at these wavelengths) and SgrA* were two separate sources. SgrA is the rbight red soiurce just to the right of the center and IRS 7 , the brightest sources at $2 \mu \mathrm{m}$, is the blue star directly above it. IRS $2 / 13$ are the pair of white sources just below right from $\mathrm{SgrA}^{*}$

(For colour plate of figure see page $\mathbf{x x}$ )

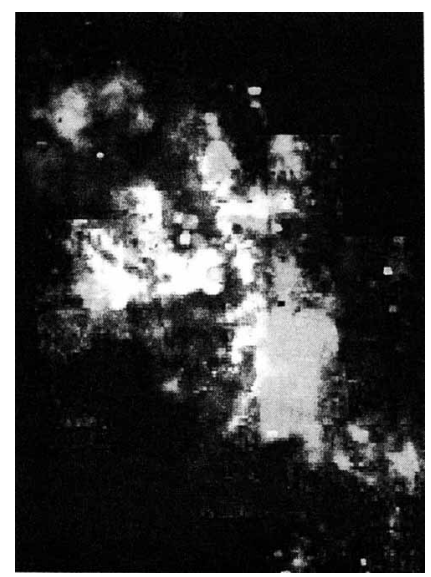

Figure An image of the hot stars and gas in the centeral 2 parsecs of the Galaxy, obtained with the method of spectroscopic drift scanning of IRIS (from [8].) Blue denotes emission from a HeI line at $2.06 \mu \mathrm{m}$, and shows a cluster of a dozen mascive stars in the nucleus. They are surrounded by the ionized ghas ofthe radio "mini-spiral", here seen in green through the ermission of hydrogen $\mathrm{Br} \gamma$ at $2.17 \mu \mathrm{m}$. Surroudning the ionized cavity is a hot clumpy molecular ring, seen in red through the light of molecular hydrogen at $2.12 \mu \boldsymbol{p}$.

(For colour plate of figure see page $\mathbf{x x}$ ) 